\title{
Cosmography: \\ Cosmology without the Einstein equations
}

\author{
Matt Visser \\ School of Mathematics, Statistics, and Computer Science, \\ Victoria University of Wellington, PO Box 600, Wellington, New Zealand \\ E-mail: matt.visser@mcs.vuw.ac.nz
}

\begin{abstract}
How much of modern cosmology is really cosmography? How much of modern cosmology is independent of the Einstein equations? (Independent of the Friedmann equations?) These questions are becoming increasingly germane - as the models cosmologists use for the stress-energy content of the universe become increasingly baroque, it behoves us to step back a little and carefully disentangle cosmological kinematics from cosmological dynamics. The use of basic symmetry principles (such as the cosmological principle) permits us to do a considerable amount, without ever having to address the vexatious issues of just how much "dark energy", "dark matter", "quintessence", and/or "phantom matter" is needed in order to satisfy the Einstein equations. This is the sub-sector of cosmology that Weinberg refers to as "cosmography", and in this article I will explore the extent to which cosmography is sufficient for analyzing the Hubble law and so describing many of the features of the universe around us.
\end{abstract}

Based on a talk presented at ACRGR4, the 4 th Australasian Conference on General Relativity and Gravitation, Monash University, Melbourne, January 2004.

To appear in the proceedings, in General Relativity and Gravitation.

Dated: 14 June 2004; IATEX-ed 6 February 2008 


\section{Introduction}

In this article I discuss a "phenomenological" approach to cosmography. Specifically I take a hard look at the question of just how much of modern cosmology can be extracted from symmetry principles and direct observation - without ever invoking the Einstein equations (Friedmann equation), and so without ever having to deal with contentious issues regarding "dark energy", "dark matter", "quintessence", and/or "phantom matter" [1].

Indeed, a surprising amount of modern cosmology is pure kinematics, what Weinberg [2] refers to as cosmography, and is completely independent of the underlying dynamics governing the evolution of the universe. For instance, it is well-known that basic symmetry principles (and in particular the cosmological principle) are sufficient to deduce the form of the cosmological metric - up to possible topological ambiguities it must be a Friedmann-Robertson-Walker [FRW] cosmology

$$
\mathrm{d} s^{2}=-c^{2} \mathrm{~d} t^{2}+a(t)^{2}\left[\frac{\mathrm{d} r^{2}}{1-k r^{2}}+r^{2}\left(\mathrm{~d} \theta^{2}+\sin ^{2} \theta \mathrm{d} \phi^{2}\right)\right] .
$$

Whereas pure cosmography by itself will not predict anything about the scale factor $a(t)$, in the cosmographic scenario we can to some extent infer the history of the scale factor $a(t)$ from the observational data while steadfastly avoiding use of the Einstein equations. In view of the many controversies currently surrounding the composition of the cosmological fluid, and the large number of speculative models presently being considered, such an observationally driven reconstruction is of interest in its own right.

\section{Hubble law}

Now in observational cosmology we do not have direct access to the complete history of the scale factor $a(t)$ over the entire age of the universe - we do however have access [however imprecise] to the current value of the scale factor and its derivatives, as encoded in the Hubble parameter, deceleration parameter, etc. This more limited information can still be used to extract useful information about the [relatively recent] history of our universe.

To set the notation as in reference [1], it is standard terminology in mechanics that the first four time derivatives of position are referred to as velocity, acceleration, jerk, and snap. Jerk [the third time derivative] is also sometimes referred to as jolt. Less common alternative terminologies are pulse, impulse, bounce, surge, shock, and super-acceleration. Snap [the fourth time derivative] is also sometimes called jounce. The fifth and sixth time derivatives are sometimes somewhat facetiously referred to as crackle and pop.

So in a cosmological setting this makes it appropriate to define Hubble, deceleration, jerk, and snap parameters as:

$$
H(t)=+\frac{1}{a} \frac{\mathrm{d} a}{\mathrm{~d} t}
$$


Cosmography: Cosmology without the Einstein equations

$$
\begin{aligned}
& q(t)=-\frac{1}{a} \frac{\mathrm{d}^{2} a}{\mathrm{~d} t^{2}}\left[\frac{1}{a} \frac{\mathrm{d} a}{\mathrm{~d} t}\right]^{-2} ; \\
& j(t)=+\frac{1}{a} \frac{\mathrm{d}^{3} a}{\mathrm{~d} t^{3}}\left[\frac{1}{a} \frac{\mathrm{d} a}{\mathrm{~d} t}\right]^{-3} ; \\
& s(t)=+\frac{1}{a} \frac{\mathrm{d}^{4} a}{\mathrm{~d} t^{4}}\left[\frac{1}{a} \frac{\mathrm{d} a}{\mathrm{~d} t}\right]^{-4} .
\end{aligned}
$$

The deceleration, jerk, and snap parameters defined in this way are dimensionless, and we can write

$$
\begin{gathered}
a(t)=a_{0}\left\{1+H_{0}\left(t-t_{0}\right)-\frac{1}{2} q_{0} H_{0}^{2}\left(t-t_{0}\right)^{2}+\frac{1}{3 !} j_{0} H_{0}^{3}\left(t-t_{0}\right)^{3}\right. \\
\left.+\frac{1}{4 !} s_{0} H_{0}^{4}\left(t-t_{0}\right)^{4}+O\left(\left[t-t_{0}\right]^{5}\right)\right\} .
\end{gathered}
$$

Now the physical distance travelled by a photon that is emitted at time $t_{*}$ and absorbed at the current epoch $t_{0}$ is

$$
D=c \int \mathrm{d} t=c\left(t_{0}-t_{*}\right),
$$

where the time difference $\Delta t=t_{0}-t_{*}$ is called the "lookback time". In terms of this physical distance travelled the Hubble law is exact but completely impractical:

$$
1+z=\frac{a\left(t_{0}\right)}{a\left(t_{*}\right)}=\frac{a\left(t_{0}\right)}{a\left(t_{0}-\Delta t\right)}=\frac{a\left(t_{0}\right)}{a\left(t_{0}-D / c\right)},
$$

A more useful result is obtained by performing a Taylor series expansion. Working to fourth order in $D$, or more precisely to fourth order in the dimensionless parameter $D H_{0} / c$, yields

$$
\begin{aligned}
\frac{a\left(t_{0}\right)}{a\left(t_{0}-D / c\right)}= & 1+\frac{H_{0} D}{c}+\frac{2+q_{0}}{2} \frac{H_{0}^{2} D^{2}}{c^{2}}+\frac{6\left(1+q_{0}\right)+j_{0}}{6} \frac{H_{0}^{3} D^{3}}{c^{3}} \\
& +\frac{24-s_{0}+8 j_{0}+36 q_{0}+6 q_{0}^{2}}{24} \frac{H_{0}^{4} D^{4}}{c^{4}}+O\left[\left(\frac{H_{0} D}{c}\right)^{5}\right] .
\end{aligned}
$$

So that

$$
\begin{aligned}
z(D)= & \frac{H_{0} D}{c}+\frac{2+q_{0}}{2} \frac{H_{0}^{2} D^{2}}{c^{2}}+\frac{6\left(1+q_{0}\right)+j_{0}}{6} \frac{H_{0}^{3} D^{3}}{c^{3}} \\
& +\frac{24-s_{0}+8 j_{0}+36 q_{0}+6 q_{0}^{2}}{24} \frac{H_{0}^{4} D^{4}}{c^{4}}+O\left[\left(\frac{H_{0} D}{c}\right)^{5}\right] .
\end{aligned}
$$

Reversion of this power series, to convert $z(D) \rightarrow D(z)$, leads to:

$$
\begin{aligned}
D(z)=\frac{c z}{H_{0}}\{1 & -\left[1+\frac{q_{0}}{2}\right] z+\left[1+q_{0}+\frac{q_{0}^{2}}{2}-\frac{j_{0}}{6}\right] z^{2} \\
& \left.-\left[1+\frac{3}{2} q_{0}\left(1+q_{0}\right)+\frac{5}{8} q_{0}^{3}-\frac{1}{2} j_{0}-\frac{5}{12} q_{0} j_{0}-\frac{s_{0}}{24}\right] z^{3}+O\left(z^{4}\right)\right\} .
\end{aligned}
$$

This simple calculation is enough to demonstrate that the jerk shows up at third order in the Hubble law, and the snap at fourth order. Generally, the $O\left(z^{n}\right)$ term in this version 
of the Hubble law will depend linearly on the $n$-th time derivative of the scale factor, and nonlinearly on lower-order time derivatives. (Also note that one of the virtues of this particular version of the Hubble law is that it is completely independent of $k$, the sign of space curvature, and is completely independent of $a_{0}$, the present-day value of the scale factor.) Carrying out fifth-order or even sixth-order expansions in terms of analogously defined crackle and pop parameters is straightforward with the aid of a symbolic algebra system such as Maple, but the formulae grow so clumsy as to be not particularly useful.

Unfortunately physical distance $D$ (or equivalently the lookback time $\Delta t$ ) is typically not the variable in terms of which the Hubble law is observationally presented. That role is more typically played by the "luminosity distance", $d_{L}$. For instance, Weinberg defines [2]

$$
\text { (energy flux) }=\frac{L}{4 \pi d_{L}^{2}}
$$

Let the photon be emitted at $r$-coordinate $r=0$ at time $t_{*}$, and absorbed at $r$-coordinate $r=r_{0}$ at time $t_{0}$. Then it is a purely geometrical textbook result that

$$
d_{L}=a\left(t_{0}\right)^{2} \frac{r_{0}}{a\left(t_{*}\right)}=\frac{a_{0}}{a\left(t_{0}-D / c\right)}\left(a_{0} r_{0}\right) .
$$

Thus to calculate $d_{L}(D)$ we need $r_{0}(D)$. A brief and quite standard computation yields

$$
r_{0}(D)=\left\{\begin{array}{cc}
\sin \left(\int_{t_{0}-D / c}^{t_{0}} \frac{c \mathrm{~d} t}{a(t)}\right) & k=+1 ; \\
\int_{t_{0}-D / c}^{t_{0}} \frac{c \mathrm{~d} t}{a(t)} & k=0 ; \\
\sinh \left(\int_{t_{0}-D / c}^{t_{0}} \frac{c \mathrm{~d} t}{a(t)}\right) & k=-1 ;
\end{array}\right.
$$

where we now must deal with the three possible signs for space curvature, $k=-1 / 0 /+1$, separately. We now Taylor series expand this result for "short" distances $D$. First note that

$r_{0}(D)=\left[\int_{t_{0}-D / c}^{t_{0}} \frac{c \mathrm{~d} t}{a(t)}\right]-\frac{k}{3 !}\left[\int_{t_{0}-D / c}^{t_{0}} \frac{c \mathrm{~d} t}{a(t)}\right]^{3}+O\left(\left[\int_{t_{0}-D / c}^{t_{0}} \frac{c \mathrm{~d} t}{a(t)}\right]^{5}\right)$,

and observe that the sign of the space curvature $k$ explicitly shows up in the third-order term. Now expand the integrals above to third order. (We can easily check, a posteriori, that this is sufficient for the final result for $d_{L}(z)$ quoted below.) Then

$$
\begin{aligned}
\int_{t_{0}-D / c}^{t_{0}} \frac{c \mathrm{~d} t}{a(t)}= & \frac{D}{a_{0}}\left\{1+\frac{1}{2} \frac{H_{0} D}{c}+\left[\frac{2+q_{0}}{6}\right]\left(\frac{H_{0} D}{c}\right)^{2}+\left[\frac{6\left(1+q_{0}\right)+j_{0}}{24}\right]\left(\frac{H_{0} D}{c}\right)^{3}\right. \\
& \left.+O\left[\left(\frac{H_{0} D}{c}\right)^{4}\right]\right\} .
\end{aligned}
$$


So we see that the conversion from $D$, the physical distance travelled, to $r$ coordinate traversed is given by

$$
\begin{aligned}
r_{0}(D)=\frac{D}{a_{0}}\{1 & +\frac{1}{2} \frac{H_{0} D}{c}+\frac{1}{6}\left[2+q_{0}-\frac{k c^{2}}{H_{0}^{2} a_{0}^{2}}\right]\left(\frac{H_{0} D}{c}\right)^{2} \\
& \left.+\frac{1}{24}\left[6\left(1+q_{0}\right)+j_{0}-6 \frac{k c^{2}}{H_{0}^{2} a_{0}^{2}}\right]\left(\frac{H_{0} D}{c}\right)^{3}+O\left[\left(\frac{H_{0} D}{c}\right)^{4}\right]\right\} .
\end{aligned}
$$

Combining these formulae we find that the luminosity distance as a function of $D$, the physical distance travelled, is:

$$
\begin{aligned}
d_{L}(D)=D\{1 & +\frac{3}{2}\left(\frac{H_{0} D}{c}\right)+\frac{1}{6}\left[11+4 q_{0}-\frac{k c^{2}}{H_{0}^{2} a_{0}^{2}}\right]\left(\frac{H_{0} D}{c}\right)^{2} \\
& \left.+\frac{1}{24}\left[50+40 q_{0}+5 j_{0}-10 \frac{k c^{2}}{H_{0}^{2} a_{0}^{2}}\right]\left(\frac{H_{0} D}{c}\right)^{3}+O\left[\left(\frac{H_{0} D}{c}\right)^{4}\right]\right\} .
\end{aligned}
$$

Now using the series expansion for for $D(z)$ we finally derive, on purely geometrical grounds, the luminosity-distance version of the Hubble law:

$$
\begin{aligned}
& d_{L}(z)=\frac{c z}{H_{0}}\left\{1+\frac{1}{2}\left[1-q_{0}\right] z-\frac{1}{6}\left[1-q_{0}-3 q_{0}^{2}+j_{0}+\frac{k c^{2}}{H_{0}^{2} a_{0}^{2}}\right] z^{2}\right. \\
&+\frac{1}{24}\left[2-2 q_{0}-15 q_{0}^{2}-15 q_{0}^{3}+5 j_{0}+10 q_{0} j_{0}+s_{0}+\frac{2 k c^{2}\left(1+3 q_{0}\right)}{H_{0}^{2} a_{0}^{2}}\right] z^{3} \\
&+\left.O\left(z^{4}\right)\right\} .
\end{aligned}
$$

The first two terms above are Weinberg's version of the Hubble law. His equation (14.6.8). The third term is equivalent to that obtained by Chiba and Nakamura [3], and by Visser [1], and depends on the jerk parameter $j_{0}$, the sign of space curvature $k$, and the present day value of the scale factor $a_{0}$. It is only at this third-order term in the Hubble law that we even begin to probe the geometry of space, and even then the fact that we are sensitive to the geometry of space depends on our choice of distance scale - recall that the physical distance Hubble law $D(z)$ as embodied in equation (12) is completely insensitive to the geometry of space (not spacetime). The fourth-order term of either the luminosity distance or the physical distance version of the Hubble law is (as expected) linearly dependent on the snap. From the derivation above it is now clear that the $O\left(z^{n}\right)$ term in this luminosity distance version of the Hubble law will also depend linearly on the $n$-th time derivative of the scale factor. It is also clear, if somewhat awkward, how to extend the calculation to arbitrarily high order in redshift.

If one instead chooses to work with angular diameter distance $d_{A}(z)$ or propermotion distance $d_{M}(z)$ the relevant conversions are straightforward [2]

$$
d_{A}(z)=\frac{d_{L}(z)}{(1+z)^{2}} ; \quad d_{M}(z)=\frac{d_{L}(z)}{1+z} .
$$

It is important to realise that any of these versions of the Hubble law, and indeed the entire discussion of this article, is completely independent of the Einstein equations 
- it assumes only that the geometry of the universe is well approximated by a FRW cosmology, but does not invoke any particular matter model. Note further that in comparison to the physical distance travelled $D(z)$ Hubble law, this luminosity distance $d_{L}(z)$ Hubble law first differs in the coefficient of the $O\left(z^{2}\right)$ term - you will still get the same Hubble parameter, but if you are not sure which definition of "distance" you are using you may mis-estimate the higher-order coefficients (deceleration, jerk, and snap).

\section{Cosmological inflation}

From a theoretical perspective, $H_{0} a_{0} / c \gg 1$ is a generic prediction of inflationary cosmology - thus assuming cosmological inflation effectively permits is to write

$$
\begin{aligned}
d_{L}(z)=\frac{c z}{H_{0}} & \left\{1+\frac{1}{2}\left[1-q_{0}\right] z-\frac{1}{6}\left[1-q_{0}-3 q_{0}^{2}+j_{0}\right] z^{2}\right. \\
& \left.+\frac{1}{24}\left[2-2 q_{0}-15 q_{0}^{2}-15 q_{0}^{3}+5 j_{0}+10 q_{0} j_{0}+s_{0}\right] z^{3}+O\left(z^{4}\right)\right\} .
\end{aligned}
$$

It is, however, important to realise that this is not the same as saying that cosmological inflation predicts $k=0$. Instead what generic cosmological inflation predicts is the much weaker statement that for all practical purposes the present day universe is indistinguishable from a $k=0$ spatially flat universe. This means that if our universe happens to be a topologically trivial $k=0$ FRW cosmology, then in a formal logical sense we will never be able to prove it. All we will ever be able to do is to place increasingly stringent lower bounds on the dimensionless parameter $H_{0} a_{0} / c$, but this will never rigorously permit us to conclude that $k=0$. The fundamental reason for this often overlooked but trivial observation is that a topologically trivial $k=0$ FRW universe can be mimicked to arbitrary accuracy by a $k= \pm 1$ FRW universe provided the scale factor is big enough. (If the universe has nontrivial spatial topology there is a possibility of using the compactification scale, which might be [but does not have to be] much smaller than the scale factor, to indirectly distinguish between $k=-1 / 0 /+1$.) In contrast if the true state of affairs is $k= \pm 1$, then with good enough data on $H_{0} a_{0}$ we will in principle be able to determine upper bounds which (at some appropriate level of statistical uncertainty) demonstrate that $k \neq 0$. Also note that even in inflationary cosmologies it is not true that $H(t) a(t) / c \gg 1$ at all times, and in particular this inequality may be violated (and very often is violated) in the pre-inflationary epoch.

\section{Discussion}

The presentation of this article now makes it clear what can and cannot be expected, even in principle, from improved observations of the luminosity distance Hubble law $d_{L}(z)$ (or for that matter its angular-distance or proper-motion distance variants). As more data is collected, at progressively higher redshifts, we can better bound the derivatives $\mathrm{d}^{n}\left[d_{L}(z)\right] /\left.\mathrm{d} z^{n}\right|_{z=0}$. This allows one in principle to extract the Hubble and deceleration parameters, but even at $O\left(z^{3}\right)$ there is a problem in that the number of 
free parameters in the Hubble law exceeds the number of measurable coefficients. This arises because of the fact that the $O\left(z^{3}\right)$ term depends explicitly on both the jerk $j_{0}$ and the scale factor $a_{0}$. This problem persists at $O\left(z^{4}\right)$ and higher, with the number of free parameters in the Hubble law always exceeding the number of measurable coefficients by one.

If (and only if) one has some independent method for bounding the scale factor $a_{0}$ (or more precisely the space curvature $k / a_{0}^{2}$ ) can one even in principle use the observational Hubble law to bound the jerk, snap, and higher time derivatives of the scale factor. One could for instance use theoretical considerations based on the assumed occurrence of cosmological inflation to effectively set $k / a_{0}^{2} \rightarrow 0$, but should then be aware that one is making a very definite additional theoretical assumption [1, 4, 5], well beyond the simple symmetry considerations of the standard FRW cosmology.

It is also worth noting that nothing in this article has made any use of the Einstein equations, or their specialization to FRW spacetimes, the Friedmann equations. Thus all comments made in this article are completely independent of one's favourite choice of matter model for the cosmological fluid. There are currently very many quite radically different models for the cosmological fluid under active consideration. Though these models often make dramatically differing predictions in the distant past (e.g., a "bounce") or future (e.g., a "big rip") there is considerable degeneracy among the models in that many physically quite different models are compatible with present day observations. Despite the fact that some parameters in cosmology are now known to high accuracy, other parameters can still only be crudely bounded [6]. In particular, published bounds on the jerk parameter are still relatively weak, and published bounds on the snap parameter are nonexistent $[1,4,5]$.

\section{Acknowledgments}

This Research was supported by the Marsden Fund administered by the Royal Society of New Zealand.

\section{References}

[1] M. Visser, "Jerk, snap, and the cosmological equation of state," Class. Quant. Grav. 21 (2004) 2603 [arXiv:gr-qc/0309109].

[2] S. Weinberg, Gravitation and cosmology: Principles and applications of the general theory of relativity, (Wiley, New York, 1972).

[3] T. Chiba and T. Nakamura, "The luminosity distance, the equation of state, and the geometry of the universe", Prog. Theor. Phys. 100 (1998) 1077 [arXiv:astro-ph/9808022].

[4] A. G. Riess et al., "Type Ia Supernova Discoveries at zi1 From the Hubble Space Telescope: Evidence for Past Deceleration and Constraints on Dark Energy Evolution," arXiv:astro$\mathrm{ph} / 0402512$.

[5] R. R. Caldwell and M. Kamionkowski, "Expansion, Geometry, and Gravity," arXiv:astro$\mathrm{ph} / 0403003$.

[6] S. L. Bridle, O. Lahav, J. P. Ostriker and P. J. Steinhardt, "Precision Cosmology? Not Just Yet", Science 299 (2003) 1532 [arXiv:astro-ph/0303180]. 